\title{
Uterine Corpus Neoplasm
}

National Cancer Institute

\section{Source}

National Cancer Institute. Uterine Corpus Neoplasm. NCI Thesaurus. Code C6300.

A benign or malignant neoplasm that affects the uterine corpus. Representative

examples of benign neoplasms include leiomyoma, adenomyoma, endometrial stromal

nodule, and endometrial polyp. Representative examples of malignant neoplasms include

endometrial carcinoma, adenosarcoma, carcinosarcoma, and leiomyosarcoma. 